\title{
OSCILLATION IN FIRST ORDER NONLINEAR RETARDED ARGUMENT DIFFERENTIAL EQUATIONS
}

\author{
WARREN E. SHREVE
}

\begin{abstract}
A result of Ladas; Lakshmikantham, and Papadakis [1] concerning oscillation caused by lag in linear first order retarded argument differential equations is generalized to the sublinear case. Examples showing that such generalization to the superlinear case is impossible are given.
\end{abstract}

0. Introduction. It is known ([1], for example) that

(A) $g \in C(R, R), g(t)<t$ for $t \in R, g(t)$ is strictly increasing on $R$ and $\lim g(t)=+\infty, t \rightarrow+\infty$,

(B) $a(t)$ locally integrable, $a(t) \geqq 0$ a.e., and

(C) $\lim \sup _{t \rightarrow \infty} \int_{g(t)}^{t} a(s) d s>1$, together imply that every solution to

$$
x^{\prime}(t)+a(t) x(g(t))=0
$$

is oscillatory, i.e. has arbitrarily large zeros. We show in this note that this result can be generalized to the sublinear case but a corresponding generalization to the superlinear case fails.

In particular we consider the more general retarded argument differential equation

$$
x^{\prime}(t)+a(t) f(x(g(t)))=0
$$

for $t \in[b,+\infty)$ where

(D) $x f(x)>0$ for $x \neq 0, f \in C(R, R), f$ is nondecreasing with $|f(x)| \rightarrow$ $+\infty$ as $|x| \rightarrow+\infty$.

We also assume $g(t)$ satisfies (A) and $a(t)$ satisfies (B).

We shall call $f$ generalized sublinear in case $\lim _{x \rightarrow 0}(x / f(x))=M<+\infty$, for some $M$. This includes the sublinear case (see [2]) $f(x)=x^{\alpha}, 0<\alpha<1$, as well as the linear case. Similarly, $f$ is generalized superlinear in case $\lim _{x \rightarrow 0}(x / f(x))=+\infty$. For simplicity we drop the word "generalized".

1. Sublinear case. We begin with a lemma.

LEMмA 1.1. For $g$ satisfying (A) and $\left\{t_{n}\right\}$ defined by $t_{0} \in R$ arbitrary and $t_{i+1}=g^{-1}\left(t_{i}\right), t_{n} \rightarrow+\infty$ as $h \rightarrow \infty$.

Received by the editors October 10, 1972 and, in revised form, March 22, 1973.

AMS (MOS) subject classifications (1970). Primary 34K15.

Key words and phrases. Retarded argument differential equation, sublinear, superlinear, oscillation of solutions.

(c) American Mathematical Society 1973 
Proof. If result is false, $t_{n} \uparrow \beta<+\infty$, and by the continuity of $g$, and, hence, $g^{-1}, \beta=\lim t_{n}=\lim g^{-1}\left(t_{n+1}\right)=g^{-1}(\beta)>\beta$. Contradiction.

We are now ready to prove a generalization of the result stated in the Introduction.

THEOREM 1. If $f$ is sublinear and satisfies (D), if

$$
\lim _{x \rightarrow 0} \frac{x}{f(x)}=M, \quad \limsup _{t \rightarrow \infty} \int_{g(t)}^{t} a(s) d s>M
$$

then every solution to (2) is oscillatory.

Proof. First suppose that $\lim \sup _{t \rightarrow \infty} \int_{g(t)}^{t} a(s) d s \geqq M+2 K$ where $K>0$. Let $x$ be a nonoscillatory solution. Then eventually $|x(t)|>0$. Without loss in generality assume $x(t)>0$ beyond $t_{0}$. Then $x^{\prime}(t)=$ $-a(t) f(x(g(t))) \leqq 0$ beyond $t_{1}=g^{-1}\left(t_{0}\right)$. Thus $x$ is nonincreasing and has a finite nonnegative limit $\alpha$ as $t \rightarrow+\infty$. Now, $\alpha=0$. For, if not, $\alpha>0$ and $f(\alpha)>0$. By the integral condition, given $t^{*}$, there is $\tau \geqq t^{*}$ with $\int_{o(\tau)}^{r} a(s) d s \geqq M+K$. Inductively define a sequence $\left\{s_{n}\right\}$ as follows. Let $s_{0}=t_{0}$ and $s_{1}=g^{-1}\left(s_{0}\right)$ such that $\int_{g\left(s_{1}\right)}^{s_{1}} a(s) d s>M+K$. Having defined $s_{n-1}$, let $s_{n}=g^{-1}\left(s_{n-1}\right)$ be such that $\int_{\substack{\left.s_{n} \\ s_{n}\right)}}^{\left.s_{(1}\right)} a(s) d s>M+K$. Then integrating we have

$$
\begin{aligned}
x\left(s_{n}\right)-x\left(s_{0}\right) & =-\int_{s_{0}}^{s_{n}} a(s) f(x(g(s))) d s \\
& \leqq-f(\alpha) \sum_{i=1}^{n} \int_{s_{i-1}}^{s_{i}} a(s) d s \leqq-f(\alpha) \sum_{i=1}^{n} \int_{g\left(s_{i}\right)}^{s_{i}} a(s) d s \\
& <-f(\alpha) n(M+K) .
\end{aligned}
$$

Thus $x\left(s_{n}\right) \rightarrow-\infty$ as $n \rightarrow \infty$ which contradicts $x(t) \geqq \alpha$ for $t \geqq s_{0}$, and $\alpha=0$ as claimed.

Beyond $g^{-1}\left(t_{1}\right), x(g(t))$ is nonincreasing and converging to zero as $t \rightarrow+\infty$. Thus, given $\varepsilon=K / 2>0$ there is a $t_{2} \geqq g^{-1}\left(t_{1}\right)$ such that $t \geqq t_{2}$ implies $x(g(t)) / f(x(g(t)))<M+K / 2$. Choose $T$ such that $g(T) \geqq t_{2}$, and $\int_{\sigma(T)}^{T} a(s) d s>M+K$. Then

$$
\begin{aligned}
x(T)-x(g(T)) & =-\int_{g(T)}^{T} a(s) f(x(g(s))) d s \\
& \leqq-\int_{g(T)}^{T} a(s) d s f(x(g(T)))<-(M+K) f(x(g(T))) \\
& <-\left(\frac{x(g(T))}{f(x(g(T)))}+\frac{K}{2}\right) f(x(g(T)))<-x(g(T)) .
\end{aligned}
$$

Thus, $x(T)<0$, contradicting its assumed nonoscillatory behavior. Therefore, $x(t)$ is an oscillatory solution. 
2. Superlinear case. The following example shows that nonoscillation is possible for $f$ superlinear even though $a(t)$ is nontrivial and satisfies a condition stronger then (C). Let $f(x)=x^{3}, a(t)=(t-\sqrt{ } t)^{3} t^{-2}$ and $g(t)=$ $t-\sqrt{ } t$. Consider the interval $[2,+\infty)$. On $I, x(t)=t^{-1}$ is a nonoscillatory solution and $\lim _{t \rightarrow+\infty} \int_{g(t)}^{t} a(s) d s=+\infty$.

Of course, if $a(t) \equiv 0$ then all solutions are eventually constant. However, such a function $a$ destroys the superlinearity of $f$. We now show that it is possible to construct a nonzero $a(t)$ for $g$ satisfying (A) and $f$ superlinear satisfying (D), such that equation (2) has an oscillatory solution. To this end we prove the following lemma.

LEMMA 2.1. Let $Z_{\alpha}$ be the first zero of the solution of (2) with initial condition $x(t) \equiv \alpha$ on $[g(b), b]$, (if no such zero exists define $Z_{\alpha}=+\infty$ ) where $g(t)$ and $a(t)$ satisfy $(\mathrm{A})$ and $(\mathrm{B})$ respectively, and fis superlinear while satisfying (D). Then $Z_{\alpha} \uparrow+\infty$ as $\alpha \downarrow 0$.

Proof. On $\left[g(b), g^{-1}\left(Z_{\alpha}\right)\right], \quad x$ is nonincreasing. Hence, $f(\alpha) \geqq$ $f(x(g(s))) \geqq 0$ for $s \in\left[b, Z_{\alpha}\right]$. Integrating, we obtain

$$
\alpha=\int_{0}^{Z_{\alpha}} a(s) f(x(g(s))) d s \leqq \int_{0}^{Z_{\alpha}} a(s) d s f(\alpha) .
$$

Thus $\alpha / f(\alpha) \leqq \int_{0}^{Z_{\alpha}} a(s) d s$, and, therefore, $Z_{\alpha} \uparrow+\infty$ as $\alpha \downarrow 0$.

This gives us an immediate nonoscillation result.

COROLlary 2.2. There is an $\alpha_{0}>0$ with $Z_{\alpha_{0}}=+\infty$ if and only if $\int_{b}^{+\infty} a(s) d s<+\infty$.

Assuming $f$ is superlinear and $g$ and $f$ satisfy (A) and (D), respectively, we now construct a piecewise continuous function $a(t)$ and a corresponding nontrivial oscillatory solution $x$ of (2). The range of $a(t)$ is $\{0,1\}$ so that $a(t)$ may be thought of as a two position control. Let $a(t)=1$ on $\left[0, g^{-1}\left(Z_{1}\right)\right]$ where, by Corollary 2.2, $Z_{1}<+\infty$ is the first zero of the solution to

$$
x^{\prime}(t)+f(x(g(t)))=0
$$

satisfying the initial condition $x(t) \equiv 1$ on $[g(0), 0]$. Define $a(t)=0$ on $\left(g^{-1}\left(Z_{1}\right), g^{-1}\left(g^{-1}\left(Z_{1}\right)\right)\right)$. Then $x(t)=\alpha_{2}<0$ for all

$$
t \in\left[g^{-1}\left(Z_{1}\right), g^{-1}\left(g^{-1}\left(Z_{1}\right)\right)\right] \equiv I_{2} .
$$

Define $Z_{2}$ to be the first zero on $\left[g^{-1}\left(g^{-1}\left(Z_{1}\right)\right),+\infty\right)$ of the solution to (3) satisfying $x(t)=\alpha_{2}$ on $I_{2}$. Define $a(t)=1$ on $\left[g^{-1}\left(g^{-1}\left(Z_{1}\right)\right), g^{-1}\left(Z_{2}\right)\right]$ and $a(t)=0$ on $\left(g^{-1}\left(Z_{2}\right), g^{-1}\left(g^{-1}\left(Z_{2}\right)\right)\right)$. Thus $x(t)=\alpha_{3}>0$ on interval $I_{3}=$ $\left[g^{-1}\left(Z_{2}\right), g^{-1}\left(g^{-1}\left(Z_{2}\right)\right)\right]$. We may generate the next zero as above. By induction we can construct a countable set of zeros, using at each step the fact that $Z_{n}<+\infty$ which follows from Corollary 2.2 for $a(t) \equiv 1$. 
It can be shown that the above solution $x(t)$ exists on $[g(0),+\infty)$ by using the strict monotonicity of $g$ to obtain $Z_{n} \rightarrow+\infty$. In view of this fact and the manner in which $a(t)$ was constructed it can further be shown that

$$
\limsup _{t \rightarrow+\infty} \int_{g(t)}^{t} a(s) d s=\limsup _{t \rightarrow+\infty}[t-g(t)] .
$$

Hence, for any $\beta \in[0,+\infty]$, by appropriate choice of $g$ one can obtain

$$
\limsup _{t \rightarrow \infty} \int_{g(t)}^{t} a(s) d s=\beta .
$$

Therefore, the oscillation of the solution $x(t)$ is independent of whether (C) holds.

\section{BIBLIOGRAPHY}

1. G. Ladas, V. Lakshmikantham and J. S. Papadakis, Oscillation of higher-order retarded differential equations generated by the retarded argument, Technical Report No. 20, University of Rhode Island, Kingston, R.I., January 1972.

2. J. W. Heidel, Oscillatory solutions for a generalized sublinear second order differential equation, Proc. Amer. Math. Soc. (to appear).

Department of Mathematics, North Dakota State University, Fargo, North DAKOTA 58102 